\title{
Plantes médicinales et populations autochtones en Guyane : perception chamanique, législation et gouvernance de la biodiversité
}

\section{Marie Fleury, Laurence Boutinot et Philippe Karpe}

\section{(2) OpenEdition \\ 1 Journals}

\section{Édition électronique}

URL : http://journals.openedition.org/elohi/804

DOI : $10.4000 /$ elohi.804

ISSN : 2268-5243

\section{Éditeur}

Presses universitaires de Bordeaux

\section{Édition imprimée}

Date de publication : 1 janvier 2014

Pagination : 189-212

ISBN : 979-10-300-0021-4

ISSN : 2431-8175

Référence électronique

Marie Fleury, Laurence Boutinot et Philippe Karpe, « Plantes médicinales et populations autochtones en Guyane : perception chamanique, législation et gouvernance de la biodiversité », ELOHI [En ligne], 5-6 | 2014, mis en ligne le 01 janvier 2015, consulté le 01 mai 2019. URL : http:// journals.openedition.org/elohi/804; DOI : 10.4000/elohi.804 


\section{Plantes médicinales et populations autochtones en Guyane : perception chamanique, législation et gouvernance de la biodiversité}

\section{MARIE FLEURY, LAURENCE BOUTINOT et PHILIPPE KARPE}

\section{Introduction}

Les plantes médicinales sont des éléments du vivant dont l'homme fait usage pour se soigner. Elles occupent donc une place particulière à l'articulation entre la nature et la culture. Leurs usages dépendent à la fois de la perception de la nature et des végétaux et aussi de la perception du corps et de la maladie. Nous proposons d'en faire ici l'analyse en Guyane, et plus particulièrement chez un des peuples autochtones, les Wayana, avec qui l'un d'entre nous travaille depuis de nombreuses années. Puis nous aborderons la manière dont la nature et, en particulier, les plantes médicinales sont perçues dans un mode de représentation occidentale, celui de la pensée scientifique. Enfin, nous étudierons la manière dont ces perceptions et représentations de la nature sont retranscrites dans les textes législatifs destinés à protéger les ressources, dans le contexte de la Convention sur la Diversité Biologique $\left(\mathrm{CDB}^{1}\right)$, et les différentes applications régionales et nationales de cette dernière.

\section{Perception animiste et totémique de la nature chez les Wayana}

Dans le mode de perception Wayana de la maladie et de la nature, on interprète certains symptômes par la rupture d'un interdit. Le chamane va, alors, prescrire

1. Traité international adopté lors du sommet de la Terre à Rio en 1992. 
des plantes pour soigner ces symptômes, dans une logique de soin qui est associée à un système de catégorisation des éléments de la nature.

\section{Les jolok ou entités spirituelles}

Chez les Wayana de Guyane, à l'instar des autres groupes amérindiens, il n'existe pas de terme générique pour désigner la nature. Toutefois, il existe une distinction lexicale entre la nature domestique et la nature sauvage. Cette distinction est soulignée dans la nomenclature botanique à travers le suffixe imë qui est ajouté pour désigner les plantes considérées comme une forme sauvage de la forme cultivée. Ce terme peut être traduit par les adjectifs " grand ", "fort ", "puissant », ou " sauvage ». En effet, chaque plante utile est supposée avoir un double « sauvage » imë plus fort, plus puissant, la plupart du temps une espèce non utilisée par l'homme, poussant dans le milieu sauvage. Cette dichotomie n'existe pas seulement dans le cas des plantes, mais également dans celui des animaux : alawata imë (le singe hurleur géant), aimala imë (l'aïmara géant), etc., sont des monstres sauvages qui apparaissent parfois dans la nature et qui ont laissé leur trace dans certains toponymes. On évoque même l'existence de Wayana imë, Palasisi imë, et Meikolo imë : " Même les Wayana, les Blancs et les Noirs ont des imë », doubles ou monstres sauvages des êtres humains que les Wayana disent apercevoir parfois. Selon les locuteurs wayana, ces monstres sauvages ne seraient pas visibles pour les Occidentaux qui nont pas la faculté de « voir» (Fleury 1999: 37).

Leur perception de la nature, qualifiée d' « animiste » par les anthropologues, donne une place particulièrement importante aux jolok, les esprits de la nature, plus ou moins puissants, en fonction des éléments concernés. "La puissance des entités naturelles suit un gradient, avec des éléments naturels dont le jolok, l'esprit, est plus ou moins élevé » (Chapuis 1998 : 808). Cette hiérarchisation des êtres naturels est associée à certaines correspondances entre plantes et animaux en fonction de critères morphologiques, analogiques, ou écologiques (Fleury $2007: 760$ ).

La puissance de la plante fait écho à un animal qui lui correspondrait dans un système de classification hiérarchique en fonction de la force de leur jolok (esprit, double spirituel).

On constate que ce système de nomenclature et de classification renvoie à une vision du monde où il n'y a pas de distinction radicale entre les humains et les espèces animales et végétales, mais où tous les êtres vivants sont hiérarchisés en fonction de la puissance de leur double spirituel. Ainsi, nous rejoignons l'analyse de Philippe Descola, qui considère que les cosmologies des Amérindiens d'Amazonie ne font pas de différence ontologique entre les humains et les 
espèces animales et végétales et que les rapports entre les humains et les non humains sont des rapports de communauté à communauté (Descola : 27).

\section{La conception chamanique de la maladie}

Dans cette perception de la nature, la maladie apparait comme un déséquilibre, une crise qui peut être engendrée par différentes causes :

- la rupture d'un interdit entraînant une maladie qui touche le fautif ou son enfant ;

- la conséquence de l'attaque d'un chamane par l'intermédiaire d'une flèche de jolok (jolok pilë);

- la conséquence d'un empoisonnement (lié à un conflit social);

- ou le vol de l'âme (omolé) par des ancêtres (Chapuis 1998 : 978).

Un désordre socio-cosmique est donc toujours à l'origine de la maladie et le rôle du chamane guérisseur va être de réparer, de restaurer l'ordre cosmique grâce à l'aide des esprits (jolok). Il est en effet le seul parmi les humains à être capable de communiquer avec eux.

Si nous prenons l'exemple de la maladie liée à la rupture d'interdit, la maladie et son traitement suivent le principe de la magie sympathique : l'homme craint de se transformer en animal par contagion directe (contact, consommation) ou indirecte (par la vue, ou le simple fait dêttre passé dans le territoire de l'animal). Le traitement consistera à utiliser une plante qui en est la correspondante végétale (Fleury $2007: 761$ ).

\section{Symptômes, thérapeutique et contexte}

naturel : une théorie des signatures ?²

Dans notre exemple, les symptômes sont interprétés comme la conséquence de la rupture d'un interdit (alimentaire ou comportemental) lié à un animal. Ces symptômes ont souvent un lien comportemental ou morphologique avec l'animal. Ainsi le lézard, dont la queue repousse après avoir été sectionnée, pourra occasionner des problèmes de croissance; le jaguar réputé pour sa voracité, entraînera des vomissements, l'enfant poussera des cris comme le toucan, la grenouille fera gonfler le ventre, etc. La maladie envoyée par l'esprit de l'animal entraîne donc un comportement proche de celui-ci, comme un début de métamorphose de lêtre humain en animal.

La plante utilisée comme remède a un lien direct avec cet animal, d’ordre organoleptique (odeur du cochon-bois, couleur de la sève rouge comme le sang)

2. Théorie formulée au $\mathrm{XvI}^{\mathrm{e}}$ siècle par Paracelse (le père de la médecine expérimentale) permettant d'assigner des correspondances entre plantes médicinales et maladies (Fleurentin et al. : 31). 
ou morphologique (liane dont les vrilles ressemblent à des griffes de lézard, feuille ressemblant à la langue du toucan). Les associations entre animaux et végétaux sont donc de type analogique, et cette analogie apparaît dans la dénomination du végétal : kiapok nu "langue de toucan", wakak omo hawin "griffes de lézard", pëinëkëpokan "odeur de pécari”, maïpuli imë "tapir géant”, etc. Parfois ces associations peuvent être d'ordre écologique, l'animal et la plante ayant le même biotope, ou plus précisément l'animal étant souvent présent à proximité de la plante, lorsqu'il consomme ses fruits ou ses fleurs par exemple. Dans ce cas également, le lien entre la plante et l'animal apparait dans la nomenclature avec le suffixe ju (Fleury $2007: 760$ ). Ce système sous-entend donc une très fine connaissance de l'environnement naturel, et un souci permanent d'en maintenir l'équilibre.

Ce mode thérapeutique fait écho à un système de classification qui établit des correspondances entre végétaux, animaux et maladies. Ce sont les jolok des animaux qui sont mis en cause dans l'interprétation de la maladie, et ce sont ceux des végétaux-remèdes qui vont intervenir dans la guérison. Le système fait donc référence aux doubles spirituels des éléments de la nature. Quand on emploie la plante correspondante à tel animal, on établit un rapport de forces entre les jolok d'animaux et ceux des végétaux. Ce système de correspondances par analogie peut également être considéré comme un système de mesure de la force intrinsèque de la plante par rapport à celle de l'animal. Ce système nous instruit donc sur une méthode d'évaluation thérapeutique des plantes, en fonction de leurs caractéristiques visibles, une sorte de "théorie des signatures", qui permettrait aux humains de décrypter la nature et les "forces intrinsèques", les jolok qu'elle sous-tend. Laction thérapeutique de la plante peut elle-même être interprétée comme une intervention spirituelle par l'intermédiaire du double spirituel de la plante, son jolok (Fleury 2007 : 761).

\section{Un système de catégorisation totémique}

Ce système de catégorisation (identification, nomenclature, référence à un ordre) nous renvoie à l'ancien système totémique de la société wayana. En effet, la société était autrefois organisée en clans à référent totémique : "les gens des fauves" kaikusijana, "les gens de l'agouti" akulijana, "les gens du tapir" maipulijana, par exemple. «Ils se nommaient par référence à un animal pour la plupart et entretenaient avec celui-ci une relation de ressemblance à travers une particularité corporelle : intonation de la voix, spécificité morphologique, coiffure, type de tatouage...» (Chapuis et Rivière : 427).

Le système de catégorisation des êtres vivants inclut, donc, tous les êtres de la nature, qu'ils soient plantes, animaux ou humains, selon un système référentiel totémique. Ce système fait écho à la période mythique des 
transformations (cf. ci-dessous), où les différentes formes de vie, animales, végétales ou humaines nétaient pas distinctes et pouvaient se transformer à volonté en l'une ou l'autre forme.

Nous avons vu que la maladie, dans certaines circonstances, peut être interprétée comme la métamorphose de l'homme en animal, en particulier lorsqu'elle est due à la rupture d'un interdit. Celui-ci est une manière de faire respecter la frontière entre les différentes formes de vie. La rupture d'interdit amorce un procédé de métamorphose de l'homme en animal que le chamane va pouvoir stopper avec un remède végétal. Pour être efficace, celui-ci doit appartenir à une catégorie faisant référence à l’animal, à son "clan totémique ". C'est l'esprit de la plante, dont la puissance doit être équivalente à celle de l'animal, qui pourra neutraliser le sort (Fleury 2007 : 761). Il en ressort une perception de la vie et de la nature où les puissances spirituelles de la nature sont incarnées dans différentes formes de vie, animale, végétale et humaine, qui peuvent être interchangeables dans certaines circonstances comme lors de la période mythique des métamorphoses (anuktatop aptau).La mythologie wayana fait souvent référence à cette période de la genèse où les différentes formes de vie nétaient pas encore figées et où tout pouvait se transformer en tout (Chapuis et Rivière : 18). De nombreuses plantes cultivées, par exemple, ont été créées à cette période, issues de la métamorphose d'un animal : c'est le cas du tabac, né de la transformation de la chenille, ou du roseau à flèche issu de celle de l'anaconda (Fleury 1999, 42). Si notre monde actuel a perdu sa fluidité et s'est figé, le temps de la genèse existe toujours et englobe notre dimension, car il est perpétuel (Chapuis et Rivière : 18).

Ce sont les puissances spirituelles, les jolok qui sont les véritables acteurs de la vie sur la terre, et qui décident de la vie, de la mort ou de la maladie des différentes formes de vie qu'ils incarnent. La perception des plantes remèdes et de leurs actions se situe donc au niveau spirituel, qui représente en réalité le vrai monde, celui qui n'est visible qu'aux yeux du chamane, mais qui régit l'équilibre de la Nature. Notre ombre, reflet de notre âme, est la preuve de l'existence de ce monde autre, de ce double spirituel dont dépend notre corps physique, matériel.

\section{Perception du monde vivant dans le mode de pensée scientifique}

À l'opposé de cette perception à la fois totémique et animiste de la nature chez les Amérindiens Wayana se situe la conception scientifique naturaliste de la théorie de lévolution de Darwin : la nature est issue de lévolution des espèces par le hasard et la nécessité des mutations génétiques (Monod). Cette vérité 
tangible et prouvée par la science cartésienne laisse peu de place aux esprits de la nature.

Bien sûr, notre propos n'est pas de mettre en cause l'une ou l'autre de ces conceptions, mais de les mettre en perspective afin d'en souligner l'influence sur la perception de la maladie et des plantes médicinales.

Une perception « naturaliste » du monde et mécanistique de la maladie

Selon Descola, c'est cette vision naturaliste du monde, propre aux sociétés occidentales, opposant la nature aux cultures, qui a permis l'apparition de la pensée scientifique moderne. Cette vision dualiste qui sous-tend toute la recherche scientifique, y compris l'anthropologie, considère la nature comme un élément extérieur, que l’on est en mesure de vouloir protéger, alors que les sociétés animistes ou totémiques, la considère comme des " voisins » avec qui elles ont des rapports sociaux (Descola : 23).

Cette perception du monde naturel s'applique également au corps humain, qui est considéré par la biomédecine $e^{3}$ comme un organisme, c'est-à-dire un ensemble d'organes ayant des interactions entre eux. Contrairement aux médecines traditionnelles, la biomédecine ne prend en compte ni le corps social (que ce soit la famille, le lignage, le clan ou la tribu), ni le corps spirituel (qu'on pourrait désigner par " âme " par facilité de langage, mais qui revêt différentes dénominations et conceptualisations selon les cultures).

Cette perception physique du corps entraîne une vision de la maladie due à une origine naturelle, concrète, représentée par un micro-organisme (virus, bactérie) ou le dérèglement d'un organe, pour une raison héréditaire, génétique ou acquise (accident). La démarche de soins qui en découle est purement mécanistique : on répare l'organe, on tue les bactéries, on neutralise les virus, on rééquilibre les composantes biologiques, on peut aussi aider l’organisme à produire des molécules pour se protéger et se soigner (immunostimulants).

On est bien loin de la conception des médecines traditionnelles où les techniques de soin vont convoquer des esprits de la nature par l'intermédiaire d'un chamane ou d'un tradipraticien ${ }^{4}$ pour donner au malade le traitement adéquat afin de rétablir l'équilibre rompu. Il est bien difficile d'évaluer l'efficacité de ces pratiques thérapeutiques traditionnelles où la puissance des

3. Médecine dont les concepts sont basés sur les principes de la biologie moderne.

4. Thérapeute exerçant une médecine reposant sur les principes d'une approche traditionnelle. 
actes symboliques et leur valeur psychothérapeutique ne peuvent être mesurées uniquement par la pharmacologie (Motte-Florac: 9).

\section{Quête de « la molécule miracle » et protection} des savoirs traditionnels

Dans la vision naturaliste du monde, la perception et l'usage des plantes médicinales suivent la même logique mécanistique : la plante va être perçue comme une usine à molécules et la recherche pharmacologique va s'attacher à décrire ces molécules, les isoler et évaluer leurs effets sur différents récepteurs (Fleurentin et al. : 50). Laction des molécules sur l'organisme humain peut être comparée à la rencontre d'une clé et d'une serrure : les cellules du corps contiennent des protéines ( «les serrures») permettant de déclencher des mécanismes. Les molécules, grâce à leur forme, vont ouvrir ou fermer la serrure, en influençant l'activité de la protéine. La recherche soriente ainsi, de plus en plus, vers la modélisation moléculaire assistée par ordinateur (Moretti et Aubertin : 40, 41). L'efficacité de la pharmacologie moderne a fait ses preuves et a permis de grandes avancées en termes thérapeutiques. C'est grâce à ces avancées dans la recherche qu'on soigne de mieux en mieux des maladies telles que le cancer, le sida et autres fléaux de notre siècle. Elle a également engendré, en recherche pharmaceutique, la quête de la molécule miracle, celle " qui donnera jour à un médicament pourvoyeur d'importantes redevances » (Aubertin 2005 : 61). En effet, les enjeux financiers sont très importants : par exemple le taxol, molécule anticancéreuse, isolée à partir de l'if du Pacifique, a servi de base à l'hémisynthèse du taxotère, un des premiers médicaments mondiaux en termes de chiffre d'affaires (Moretti et Aubertin : 32).

Ce contraste entre la vision scientifique d'un côté et, de l'autre, la vision autochtone, qui tient compte des différentes interactions entre plantes, animaux, et cosmos, a engendré des débats conflictuels entre les protagonistes des sociétés industrielles et ceux des sociétés traditionnelles, les premiers étant accusés de faire du profit au détriment des autres. Bien plus encore que le contenu factuel des savoirs traditionnels des peuples autochtones, cest toute une conception de la connaissance qui est en question et qui distingue les sociétés occidentales des autres.

Nous avons tous en mémoire des exemples de dépôt de brevet abusifs sur des plantes connues et utilisées depuis des temps immémoriaux par des populations amérindiennes : l'exemple de l'Ayahuasca est particulièrement marquant. Ce mélange de plantes (Banisteriopsis caapi et Psychotria viridis) est utilisé par les chamanes d'Amazonie à des fins initiatiques ou thérapeutiques. Or, un citoyen américain, prétendant avoir découvert une 
nouvelle variété de Banisteriopsis, déposa un brevet dans le but d'en tirer des médicaments psychiatriques. La COICA (Coordination des Organisations Indigènes du Bassin Amazonien) porta plainte et gagna le procès, ce qui entraîna l'annulation du brevet en 1999 (GRAIN 20005). Cet exemple est particulièrement significatif, car il oppose deux logiques profondément différentes : la plante et son usage sont considérés comme sacrés chez les populations indigènes tandis que le droit américain, donnant la possibilité de breveter le vivant, permettrait une appropriation privée pour en tirer des bénéfices financiers. Les brevets sur le vivant englobent, désormais, jusqu'aux séquences d'ADN humain. On pourrait citer de nombreux exemples où les populations se sont senties, à juste titre, spoliées de leurs connaissances traditionnelles pour des objectifs commerciaux, non seulement dans les pays de l'Amazonie - brevet de Pod-Ners sur le Phaseolus vulgaris, haricot mexicain (Thomas : 833) -, mais aussi en Inde ou le $\mathrm{Neem}^{6}$ (arbre dont les qualités de fongicide sont reconnues) a fait l'objet d'un brevet déposé par W. Grace, ou encore une variété de riz Basmati (Thomas, idem). De nombreux cas dans le Pacifique sont également éloquents - le mamala, arbre médicinal ${ }^{7}$; la noix de coco ou encore le kava ${ }^{8}$, une plante qui entre dans des rituels sacrés -, quand ils ne touchent pas, de surcroît, les gènes humains - gênes du diabète chez les populations Tonga ; xénotransplantation chez les populations des Îles Cook (Ratuva : 186).

C'est ainsi qu'est apparue la nécessité d'une protection juridique des savoirs traditionnels, laquelle a engendré de longs et vifs débats internationaux dans les conventions sur la diversité biologique. Du fait de la grande variété de définitions des savoirs traditionnels, selon les types de connaissances factuelles, les détenteurs de celles-ci, les modes d'acquisition et de transmission (Houde), ces savoirs écologiques relèvent de registres multiples. Il est, ainsi, difficile de limiter leur définition dans un seul champ de reconnaissance puisqu'ils concernent aussi bien des savoirs empiriques, factuels, que des modes de gestion. Ils sont liés à des contextes très localisés, représentent des valeurs éthiques de respect du vivant (Johnson ; Kuhn et Duerden ; Nadasdy ; Simpson ; Stevenson ; Callaway ; Wenzel ; Lewis

5. « GRAIN est une petite organisation internationale à but non lucratif qui soutient la lutte des paysans et des mouvements sociaux pour renforcer le contrôle des communautés sur des systèmes alimentaires fondés sur la biodiversité ", voir http://www.grain. org/fr/pages/organisation, consulté le 15 octobre 2014 (note des éditeurs).

6. Azadirachta indica.

7. Homolanthus nutans.

8. Piper methysticum. 
et Sheppard, cités par Houde ${ }^{9}$ ). Ils sont, également, vecteurs de l'identité culturelle des peuples qui les détiennent. Enfin, ils sont des façons de voir le monde, de considérer les rapports entre Humains et non Humains, et avec le cosmos (Mailhot 1993 ; cité par Houde ${ }^{10}$ ), lesquels rapports influencent directement le mode de vie de ces peuples, leur subsistance et leur gestion de l'environnement (Berkes 1988 : 11). Aussi, de nombreux auteurs s'accordent à dire que la différence entre les savoirs scientifiques occidentaux et ceux des peuples autochtones a plus à voir avec l'hégémonie du pouvoir de ceux qui imposent leur science qu'avec une "vérité " (Watson-Verran et Turnbull, cités par Houde ${ }^{11}$ ).

Le problème se situe dans les relations de pouvoir qui conduisent à l'imposition d'un système de connaissance (Nadasdy, cité par Houde) sur un autre et, partant, aux questions de gouvernance au niveau international, sur lesquelles nous reviendrons plus loin. Aussi, bien que l'on soit parvenu à un consensus sur la nécessité de protéger les droits de propriété intellectuelle des sociétés autochtones et locales, la difficulté reste de traduire en textes juridiques cette protection de savoirs collectifs contre des appropriations commerciales extérieures dont les bénéfices leur échappent.

9. Donald. G. Callaway. «Landscapes of Tradition, Landscapes of Resistance ». Northern Ethnographic Landscapes: Perspectives from Circumpolar Nations. Igor Krupnik, Rachel Mason, Tonia Horton (sous la dir. de). Washington D.C. : Smithsonian Institution (Arctic Studies Center) and National Parks Service, 2004 : 177-201 ; Martha Johnson. « Dene Traditional Knowledge ». Northern Perspectives, 20 : 1(1992) ; Richard G. Kuhn, Franck Duerden. « A Review of Traditional Environmental Knowledge : an Interdisciplinary Canadian Perspective ». Culture, 16 :1 (1996) : 71-84 ; John L. Lewis, Stephen R. J. Sheppard." Ancient Values, New Challenges : Indigenous Spiritual Perceptions of Landscapes and Forest Management ». Society and Natural Resources, $18: 10$ (2005) : 907-920 ; Paul Nadasdy." The Politics of TEK : Power and "Integration" of Knowledge ». Arctic Anthropology, 36 :1-2 (1999) : 1-18 ; Gillian Rose. Feminism and Geography: the Limits of Geographical Knowledge. Minneapolis : University of Minnesota Press, 1993 ; Leanne Betasamosake Simpson. « Aboriginal Peoples and Knowledge : Decolonizing our Processes ». Canadian Journal of Native Studies, $21: 1$ (2001) : 137-148 ; Marc G. Stevenson. «Indigenous Knowledge in Environmental Assessment ». Arctic, 49 :3(1996) : 278-291 ; George W. Wenzel. "From TEK to IQ : Inuit Qaujimajatuqangit and Inuit Cultural Ecology». Arctic Anthropology, $41: 2$ (2004) : 238-250. 10. José Mailhot. Traditional Ecological Knowledge : the Diversity of Knowledge Systems and their Study. Great Whale Environmental Assessment. Montréal : Great Whale Public Review Support Office, coll. « Background paper n 4 », 1993.

11. Helen Watson-Verran, David Turnbull." Science and Other Knowledge Systems ". Handbook of Science and Technology Studies. Sheila Jasanoff, Gerald E. Markle, James C. Petersen, Trevor Pinch (sous la dir. de). Thousand Oaks : Sage Publications, 1995 : 115-139. 
Comment faire en sorte de respecter les points de vue des Autochtones sans les instrumentaliser à travers des concepts issus des sociétés industrielles ? Certains auteurs (notamment Rose 1993 et Stevenson 1996, cités par Houde) soulignent, en effet, qu'il existe bien un paradoxe selon lequel les populations indigènes ou autochtones, notamment les Premières Nations, appuient leur légitimité, dans les négociations internationales, en réitérant le caractère traditionnel de leurs savoirs écologiques - sous-entendu relevant du passé, voire de temps immémoriaux -, face aux modes de gestion inspirés des sciences occidentales. Ce sont pourtant bien ces caractères immuables, anciens, traditionnels, statiques, folkloriques qui tendent à marginaliser les peuples autochtones au sein de la société nationale et, partant, ne renforcent pas leur pouvoir, voire invalident la légitimité de leur participation aux négociations pour la reconnaissance de leurs droits (idem).

\section{Perception du vivant dans les textes législatifs : une marchandisa- tion de la nature et des savoirs traditionnels}

\section{La vision générale des textes de référence}

Le mode de représentation de la nature dans les textes est directement lié à notre pensée scientifique naturaliste : chose, bien ou objet, la nature y est perçue par le biais de sa biodiversité, renvoyant à un inventaire du nombre d'espèces (ou de milieux) qui ne cesse de diminuer sous la pression des activités humaines, et qu'il convient de protéger, pour un usage réel ou potentiel. Cette vision, avant tout utilitariste, entraîne une évaluation économique de la nature. Celle-ci est perçue comme source potentielle de revenus ou, a mini$m a$, de services rendus à l'homme - tels, aujourd'hui, les services environnementaux : séquestration du carbone, maintien de la biodiversité, protection de bassins-versants ou de la beauté du paysage (voir Wunder).

Cette vision utilitariste entraîne une évaluation des éléments de la diversité en fonction des services rendus, et une marchandisation de la biodiversité. La difficulté, en amont, de déterminer la valeur économique des ressources naturelles, génétiques voire des services environnementaux, implique que s'instaure, du côté des peuples autochtones et des communautés locales des pays fournisseurs de ces ressources, un climat de suspicion tout à fait compréhensible. Les différents traités qui ont suivi la convention de 1992 n’ont fait que renforcer cette tendance mercantile, qu'ont bien du mal à contrecarrer les organisations indigènes souhaitant défendre leur vision du monde (Aubertin, Pinton et Boisvert : 18-19). 


\section{Des moyens de protection?}

Dès 1992, la Convention sur la Diversité Biologique reconnaît les savoirs traditionnels comme étant garants de la protection de la nature. Ainsi, dans son article 8 , paragraphe $\mathrm{j}$, la Convention stipule que chaque partie contractante « respecte, préserve et maintient les connaissances, innovations et pratiques des communautés autochtones et locales qui incarnent des modes de vie traditionnels, présentant un intérêt pour la conservation et l'utilisation durable de la diversité biologique $»^{12}$. Mais l'enjeu de la protection des savoirs traditionnels est aussi économique. Nous l'avons $\mathrm{vu}$, ces savoirs sont potentiellement utilisables par l'industrie pharmaceutique ou cosmétique à des fins commerciales.

Pour protéger les populations locales du "biopiratage " (appropriation illégitime des ressources de la biodiversité et des connaissances traditionnelles autochtones qui peuvent y être associées), les articles $8, \S \mathrm{j}$ et 15 de la CDB posent deux principes fondamentaux : le consentement préalable et informé pour l'accès aux ressources génétiques, et le partage des avantages. Ces principes trouveront un écho dans la Déclaration des Nations Unies sur les droits des peuples autochtones de 2007, et se verront approfondis, ensuite, dans le Protocole de Nagoya (Greiber et al.). Il s'agit d'encadrer la bioprospection qui recense les molécules du vivant en termes de potentialités médicale, cosmétique ou biotechnologique, et particulièrement celle qui est ethnoguidée, c'est-à-dire qui s'appuie sur des connaissances traditionnelles. En définissant les règles d’accès à la biodiversité, et en partageant les bénéfices, on reconnaît des droits de propriété des peuples autochtones sur les ressources (Thomas : 826), ce faisant, on espère restaurer la qualité de "sujet » des Autochtones, rétablir une relation égale et au final mettre fin au pillage par les pays du Nord (qui sont les plus grands détenteurs de biotechnologies) des pays du Sud (qui sont les plus riches en termes de biodiversité et de savoirs traditionnels).

Les Autochtones critiquent sévèrement les termes actuels du régime juridique de l'ABS (Access and Benefit Sharing) ou APA en français (Accès et Partage des Avantages). Citons, par exemple, la Déclaration conjointe des organismes de l'Amérique du Nord autochtones, rendue publique le 14 décembre 2010, sur le protocole ABS de Nagoya de la Convention sur la Biodiversité :

12. Voir https://www.cbd.int/doc/legal/cbd-fr.pdf, consulté le 15 octobre 2014 (note des éditeurs). 
Femmes Autochtones du Québec Inc. ${ }^{13}$ ont de sérieuses préoccupations au sujet de l'issue de la réunion d'octobre à Nagoya pour COP-1014 - en relation avec le Protocole de Nagoya sur l'accès et le partage des avantages. L'accord sur le protocole de Nagoya a été précipité dans le but de respecter léchéance fixée à COP-9, 2 ans plus tôt. Cependant, il néglige de prendre en compte les autres objectifs de la Convention sur la Diversité Biologique (CDB), principalement de protéger la biodiversité et d'assurer son utilisation durable. Le protocole de Nagoya, récemment adopté, a ses faiblesses. Il ne parvient pas à répondre aux exigences minimales requises pour prévenir la biopiraterie et il ne soutient pas, non plus, les droits collectifs des peuples autochtones. Le protocole manque de répondre aux demandes des peuples autochtones pour la pleine reconnaissance et l'application de leurs droits, qui, après 30 ans de négociations au sein de l'ONU, sont maintenant reconnus dans la Déclaration des Nations Unies sur les droits des peuples autochtones $(D N U D P A)^{15}$.

Tout en le critiquant, ils en reconnaissent aussi les qualités et en promeuvent l'usage. Ils prennent néanmoins le soin de rappeler le cadre dans lequel il doit être compris et employé, spécialement les droits reconnus dans la Déclaration des Nations Unies sur les droits des peuples autochtones, et d'en suggérer des améliorations (Hunka et Mc Neely). Mais ce statut ne peut être véritablement protecteur qu’à la condition dềtre compris et maîtrisé par les bénéficiaires, spécialement en tant qu'outil juridique (Jonas et al.). Il " ne fait [en réalité] qu’enregistrer un accord commercial et industriel d'utilisation des ressources génétiques » dans lequel " les savoirs traditionnels faisant partie du domaine public ne sont pas considérés, mais renvoyés à la législation nationale ou à l'OMPI ${ }^{16}$ "( $\mathrm{Au}$ bertin et Filoche : 10). Ainsi, ces savoirs ne sont pas considérés comme détenus par les communautés locales. Ces dernières ne peuvent donc pas revendiquer ni exercer de droits spécifiques conformes à leurs intérêts ou leur culture. Ces populations ne préservent leurs droits qu'en ayant recours aux seules règles du droit commun applicable à tous, lequel n'est

13. Association de défense qui siège à l'Assemblée des Premières Nations du Québec et du Labrador, voir http://www.faq-qnw.org/, consulté le 15 octobre 2014 (note des éditeurs).

14. $10^{\mathrm{e}}$ Conférence des Parties à la Convention sur la diversité biologique, dite COP-10. À suivre, «COP- 9 » $9^{\text {e }}$ Conférence, etc.

15. Le texte est disponible sur le site Internet : http://www.faq-qnw.org/old/documents/comuniquedepresse-14dec.pdf. Document consulté, la dernière fois, le 07 octobre 2014.

16. Organisation Mondiale de la Propriété Intellectuelle. Il s'agit d'une institution dépendant de l'ONU, voir http://www.wipo.int/about-wipo/fr/index.html, site consulté le 17 octobre 2014 (note des éditeurs). 
pas pleinement et efficacement protecteur des droits des communautés locales (Karpe) ${ }^{17}$.

\section{Le régime CDB-ADPIC-APA}

Comme le dit Frédéric Thomas, l'histoire des protocoles de brevetabilité du vivant est ancienne et a vu se faire et défaire des alliances Nord-Sud, Sud-Sud et, au final adopter le droit de souveraineté nationale sur les ressources génétiques et le vivant au détriment, de fait, de leur statut de bien communs de l'humanité, ainsi que l'ont soutenu longtemps aussi bien les pays en développement que la FAO. Lémergence des pays dits " mégadivers » (Inde, Brésil, pays andins) ${ }^{18}$ n'est pas étrangère à ce choix puisqu'ils ont compris qu'ils avaient la possibilité de valoriser leurs richesses et de faire, à terme et à leur tour, leur entrée dans les mécanismes de brevetabilité du vivant, à l'instar des pays occidentaux. Le statut de bien commun de l'humanité entravait le libre-échange des ressources génétiques et c'est pourquoi ce choix du régime de souveraineté nationale a rencontré les intérêts des industries biotechnologiques du Nord lors du Sommet de Rio en 1992. La conservation de la diversité biologique est associée, depuis lors, à la mise en valeur de ces mêmes ressources et la CDB entérine ce choix en y ajoutant la nécessité de reconnaître les savoirs écologiques traditionnels des peuples qui contribuent au maintien de la biodiversité (art. $8, \S \mathrm{j}$, précédemment cité). Cette reconnaissance des droits de propriété (DPI) va trouver un écho au sein de l'OMC à travers les ADPIC (Aspects des Droits de Propriété Intellectuelle qui touchent au Commerce). Or, il est écrit dans l'article $27.3 \mathrm{~b}$ des ADPIC que l'OMC peut « exclure de la brevetabilité les végétaux et les animaux [...] à condition de prévoir un système sui generis efficace de protection de ces variétés » (ADPIC, 1994, art. cité par Thomas : 836). Ce système laisse

17. La protection accordée par le droit commun avait encore été amoindrie par l'ajout du terme «approbation» dans le Protocole de Nagoya : il n'y avait dès lors plus besoin d'un consentement des communautés locales pour accéder à et utiliser leurs savoirs et les ressources qui leur sont liées. Après de vives critiques, spécialement des autochtones et de leurs défenseurs, la Conférence des Parties de la CBD a conféré, « de manière invariable et consensuelle » dans ses récentes décisions, le sens de " consentement préalable, libre et éclairé » au terme " approbation » (Instance permanente sur les questions autochtones. Neuvième session, New York, 19-30 avril 2010. Informations communiquées par les organismes des Nations Unies et autres organismes intergouvernementaux. Secrétariat de la Convention sur la Diversité Biologique. Document E/C.19/2010/3, $\$$ 39. Voir http://www.un.org/esa/socdev/unpfii/documents/E_2010_43_FR.pdf, site consulté le 17 octobre 2014.

18. D’après Thomas (2006: 832), le terme « mégadivers » provient du « jargon internationaliste » et désigne ainsi les pays de l'hémisphère sud qui détiennent $90 \%$ de la biodiversité mondiale. 
les États libres de ne pas reconnaître de brevet sur certaines espèces considérées vitales (idem) ainsi que l'ont fait les pays de l'Organisation de l'Unité Africaine qui refusent le principe d'application des droits de propriété intellectuelle sur le vivant (Aubertin). Mais on peut alors se demander, avec Frédéric Thomas, comment les communautés locales pourraient exiger le partage des avantages si elles refusent la brevetabilité sur le vivant.

Mais si ce système ADPIC-CDB conditionne les dépôts de brevet au mécanisme de consentement préalable et informé des populations et de partage des avantages, il est aussi un piège pour les pays mégadivers « qui n’ont d'autre choix, aujourd'hui, que de faire tomber les connaissances naturalistes de leurs populations dans le domaine public pour se prémunir de brevets futurs iniques, l'autre solution restant de passer des contrats de bioprospection avec les multinationales» (Thomas : 837). La rencontre de ces protocoles avec les exigences de la $\mathrm{CDB}$ a pour conséquence de reléguer les savoirs écologiques traditionnels au même statut de connaissances soumises à marchandisation ou à publication.

\section{CLIP ${ }^{19}$ et APA dans le cadre spécifique de la Guyane française}

En Guyane, depuis la mise en place du parc national en 2007, basée sur la nouvelle loi des parcs nationaux de 2006, une réglementation d'accès aux ressources génétiques a été instaurée en zone coeur du parc (Article L331-15-6 du code de lenvironnement ${ }^{20}$ ), nécessitant une demande d'autorisation auprès des autorités du Parc, qui la soumettent au Conseil Régional, lequel prend lui-même avis du Conseil Général.

Un document intitulé "Code de bonne conduite » a été proposé par le Conseil Scientifique du Parc Amazonien de Guyane (PAG) prévoyant, selon les termes communément admis en la matière, une demande d'autorisation libre et éclairée auprès des autorités coutumières et une restitution des résultats. Ce code de bonne conduite doit obliger à plus de transparence dans la mise en place des programmes scientifiques, et de leurs retombées. «En cas d'utilisation de connaissances traditionnelles détenues par les communautés d'habitants, le contrat s'effectue avec la consultation étroite de ces communautés et mentionne, dans une clause spécifique, les actions qui seront réalisées par la Région, visant à faire bénéficier la ou les communautés concernées des avantages découlant de l'utilisation de leurs connaissances traditionnelles " (Parc Amazonien de Guyane, 158).

19. Consentement Libre, Informé, Préalable.

20. Sauf indication contraire, toutes les références légales portent sur ce règlement actuellement en vigueur. Le Code de l'environnement peut être librement consulté sur le site Internet : http://www.legifrance.gouv.fr. 
Par ailleurs, le Congrès des élus (réunissant les Conseils Régional et Général) du 21 juillet 2011 a élaboré un texte qui donne les principales orientations proposées par les élus guyanais en termes d'accès aux ressources et partage des avantages : la Région Guyane y demande à être habilitée par le législateur à définir les règles d'APA (Accès aux ressources génétiques et Partage des Avantages) qui devront s'appliquer sur l'ensemble du territoire. En revanche, le consentement préalable des populations autochtones et locales n'est pas proposé dans le texte qui exige une simple consultation, mettant en avant leur absence de statut. Parallèlement, il demande aussi à la France de ratifier la convention 169 de l'Organisation Internationale du Travail (OIT) qui traite spécifiquement des droits des peuples indigènes (Congrès des élus de Guyane, 2011).

En effet, la France ne reconnaît pas de statut particulier aux peuples autochtones, car ce serait contraire à l'article 1 de sa Constitution. Cette interprétation peut toutefois être contestée (Karpe : 141 et suivantes). Au final, les débats qui ont eu lieu pendant lécriture de la charte du Parc national ont fini par donner raison aux représentants des populations locales dans le conseil d'administration qui ont obtenu le consentement préalable, mais sans qu’en soient précisées les modalités d'application.

Il existe, aujourd'hui, un projet de loi sur la biodiversité qui devrait voir le jour dans les mois à venir. La version ici présentée est celle du projet de loi tel qu'il a été déposé le 26 mars 2014 à l'Assemblée nationale (Projet de loi relatif à la biodiversité, $\mathrm{n}^{\circ} 1847^{21}$ ). En effet, à la date de rédaction du présent article (juin 2014), le débat sur ce texte vient à peine de débuter au Parlement. Il s'agit donc d'une version provisoire.

Son titre IV concerne l'accès aux ressources et le partage des avantages. Il a pour objet la mise en application du protocole de Nagoya, signé par la France le 20 septembre 2011, mais non encore ratifié. Il crée ainsi, notamment, une nouvelle section III au chapitre II du titre I ${ }^{\text {er }}$ du livre IV du Code de l'environnement, ainsi rédigée : "Accès aux ressources génétiques et aux connaissances traditionnelles associées, et partage des avantages découlant de leur utilisation ».

La France a choisi de mettre en place un régime d'accès APA. Le principe de souveraineté de l'État sur les ressources biologiques y est souligné, impliquant sa responsabilité eu égard à sa protection et à sa gestion. Ce projet de loi souligne, également, les droits des communautés d'habitants sur les connaissances traditionnelles associées aux ressources génétiques. Ces communautés d'habitants sont, pour la première fois, définies en droit français. Il s'agit de «toute communauté d'habitants qui tire traditionnellement ses moyens de subsistance

21.Disponible sur le site Internet : http://www.assemblee-nationale.fr/14/projets/ pl1847.asp. Document consulté, la dernière fois, le 07 octobre 2014. 
du milieu naturel et dont le mode de vie présente un intérêt pour la conservation et l'utilisation durable de la biodiversité » (nouvel article L. 412-3-4o proposé2 $^{2}$ ).

Cette définition concerne, en Guyane, principalement les populations forestières du sud du département et ainsi, exclut la plupart des communautés vivant sur le littoral, en particulier les populations créoles qui ne tirent plus, à proprement parler, leurs moyens de subsistance du milieu naturel. Ce terme de " communautés d'habitants " pose, donc, de nouveaux problèmes en termes de définition et de partage. Le terme de population autochtone est donc soigneusement évité, et les communautés sont définies par leur mode de vie " traditionnel » et la pertinence de celui-ci, ce qui promet d'engendrer de nombreux débats mais aussi d’ouvrir de nouvelles opportunités quant à une définition à venir de la nature qui exclurait sa chosification.

En termes de partage, l'État français a choisi de privilégier le retour des avantages vers la biodiversité. En effet, suivant le nouvel article L. 412-3-3ํ proposé, le partage des avantages peut consister en :

a. l'enrichissement ou la préservation de la biodiversité in situ ou ex situ;

b. la préservation des connaissances traditionnelles associées aux ressources génétiques ;

c. la contribution au développement local de filières associées à l'utilisation durable des ressources génétiques ou des connaissances traditionnelles associées, en lien avec les territoires qui ont contribué à la conservation de ces ressources;

d. la collaboration, coopération ou contribution à des activités de recherche, d'éducation, de formation, de transfert de compétences ou de transfert de technologies;

e. le versement de contributions monétaires.

Les connaissances traditionnelles sont elles-mêmes définies comme étant " les connaissances et pratiques relatives aux propriétés génétiques ou biochimiques de cette ressource, à son usage ou à ses caractéristiques, et qui sont détenues de manière ancienne et continue par une ou plusieurs communautés d'habitants [...], ainsi que les évolutions de ces connaissances et pratiques lorsqu'elles sont le fait de ces communautés d'habitants » (nouvel article L. 412$3-5^{\circ}$ proposé). Cette définition est similaire à celle employée dans le cadre de la protection du droit au patrimoine des autochtones (Karpe : 568 et suivantes).

Lexposé des motifs du projet de loi précise que « le titre IV vise à faciliter l'accès aux ressources génétiques et connaissances traditionnelles associées pour

22. Il est d'usage d'intituler les nouvelles propositions d'articles non encore définitivement adoptées et appliquées de la manière suivante : «nouvel article XXXX proposé », de manière à les distinguer facilement des articles en vigueur. 
les 'utilisateurs' (chercheurs, entreprises), en clarifiant les attentes des 'fournisseurs' (Nation en ce qui concerne les ressources génétiques, communautés d'habitants en ce qui concerne les connaissances traditionnelles associées). Cette sécurité juridique accrue contribuera au maintien d'un dynamisme d'innovation et de partenariats pérennes bénéficiant à l'ensemble des acteurs » («Exposé des motifs », page 16).

Enfin, le nouvel article L. 412-15 proposé reconnait aux collectivités d’outremer, relevant de l'article 73 de la Constitution, le droit d'exercer "à leur demande les fonctions de l'autorité administrative compétente » (idem, page 22) concernant les procédures déclaratives et d'autorisation. Ce nouveau droit permet, ainsi, de « concilier l'application uniforme de la loi nationale [...] et les demandes de certaines collectivités d’outre-mer, très impliquées dans la préservation de la biodiversité, de jouer un rôle important dans ces procédures » (idem).

\section{Le partage des avantages, une question de gouvernance?}

Si nous revenons à notre exemple qui introduisait cet article, chez les Wayana, en Guyane, le chamane obtient les remèdes pour soigner le malade, par l'intermédiaire des esprits, lors de ses séances de soins. Les savoirs traditionnels sont donc considérés comme des savoirs spirituels et sacrés, " inspirés " lors de rêves ou de transes et le chamane n'est qu'un dépositaire de ces savoirs, qui lui sont transmis par les entités spirituelles. Les plantes elles-mêmes sont des médiatrices dans une forme de dialogue entre ces entités et les Humains. La protection de ces savoirs traditionnels s'avère donc complexe à envisager d'un point de vue juridique.

En effet, la protection de la propriété intellectuelle, qui s'articule avec la liberté du commerce et de l'industrie, repose sur deux fondements principaux :

- un fondement moral, qui exige une reconnaissance morale et matérielle de la qualité d'auteur d'un créateur ;

- un fondement économique car, en garantissant une exclusivité et en assurant la loyauté dans les relations industrielles et commerciales, l'État favorise la promotion de l'exploitation des créations.

«Or les peuples autochtones ne considèrent pas leur patrimoine du point de vue de la propriété - en d'autres termes, comme un bien dont ils sont propriétaires et qui est utilisé pour en tirer des avantages économiques - mais du point de vue de la communauté et de la responsabilité individuelle " (Fleury et al :205).

Une législation qui transforme les connaissances traditionnelles en sources de revenus et qui nécessite d'identifier leur créateur risque d’entraîner une 
déviance sur la manière de les considérer. "Les savoirs naturalistes, chez les Amérindiens d'Amazonie, participent d'une praxis globale, dont la finalité est avant tout la reproduction de la société » (Descola cité par Pinton et Grenand : $172^{23}$ ). Et contrairement à certaines idées reçues, les Amérindiens d'Amazonie ne sont pas, à proprement parler, des "protecteurs de l'environnement », concept qu'ils ignorent. En revanche, leurs pratiques et leurs représentations visent à restaurer, pour le moins, l'abondance des ressources. Cependant une modification des modalités de production et de répartition des savoirs peut entraîner l'effondrement de leur culture, comme cela a déjà été le cas par le passé (Pinton et Grenand : 173).

Soulignons que dans la Loi No 2000-1207 du 13 décembre 2000 (Journal Officiel du 14 décembre 2000), l'article 33 prévoit que l'État et les collectivités locales encouragent le respect, la protection et le maintien des connaissances, innovations et pratiques des communautés autochtones et locales fondées sur leurs modes de vie traditionnels et qui contribuent à la conservation du milieu naturel et à l'usage durable de la diversité biologique.

Or, nous constatons que, malgré les efforts des différents acteurs à reconnaître des droits aux sociétés traditionnelles sur leurs propres connaissances, et la nécessité indiscutable d'un juste retour des avantages de leur exploitation commerciale vers les producteurs de ces connaissances, le fait même détablir des lois régissant les échanges risque d'entraver la dynamique de la production de ces savoirs, comme nous allons le voir ci-après.

Bien qu'un cadrage soit nécessaire, pour éviter les abus et les profits des industriels à partir des connaissances ancestrales des populations traditionnelles, il ne faudrait pas, pour autant, détruire l'essence même de ces savoirs, en les monnayant. Du point de vue politique, et au-delà même de la seule question autochtone, les droits des peuples et des nations doivent contenir le mouvement de privatisation des biens publics qui se profile à travers la brevetabilité du vivant. « La CDB est de ce point de vue là un 'poids lourd' de la coordination internationale pour l'environnement» (Louafi : 162).

En effet, dans les rapports de force entre les pays du Nord (forts de leur développement des biotechnologies et de leur capacité à déposer des brevets sur le vivant), les pays émergents (qui espèrent tirer profit, à terme, de ces nouvelles procédures de brevetabilité étant donné qu'ils possèdent également les richesses de la biodiversité) et les pays les moins avancés (comme ceux du continent africain, qui nont guère de possibilité de développer des biotechnologies), il existe des dissymétries importantes qui grèvent les possibilités

23. Philippe Descola. In the Society of Nature : a Native Ecology in Amazonia. Cambridge : Cambridge University Press, 1996. 
d'une harmonisation des procédures, ou simplement une gouvernance internationale (Thomas : 835).

Les peuples et communautés autochtones, au sein des Mégadivers, en disposant du recours du consentement libre, informé et préalable, sont en mesure de contrôler physiquement l'accès à leur territoire des bioprospecteurs afin d'éviter les biopirateries. Ce recours "serait une base suffisante " (Filoche : 452), pour que les communautés autochtones soient mieux impliquées dans les négociations sur le partage des avantages. Si le respect du consentement libre, informé, préalable (CLIP) et celui du partage des avantages (APA) préconisés par la CDB sont associés avec difficulté aux ADPIC (voir ci-dessus, "Le régime CDB-ADPIC-APA ») et aux mécanismes de brevetabilité du vivant, il reste que les négociations se poursuivent et que les enjeux de gouvernance apparaissent, dès lors, de première importance.

Les acteurs en jeu sont nombreux et ne concernent pas seulement les multinationales des biotechnologies, les centres de recherche et les États, mais aussi toute une nébuleuse d'institutions de régulation, des associations de malades, des lobbies environnementalistes, indigénistes, et de nombreuses ONGs, voire des partis politiques (Louafi : 171). Dès lors les bases contractuelles à partir desquelles se formulent les brevets et les droits commerciaux y afférant apparaissent insuffisants. Les contrats ont, désormais, évolué et sont de plus en plus sophistiqués, quand bien même ils présentent toujours des « insuffisances notables » (Louafi : 164). En 2002, la $6^{\mathrm{e}}$ Conférence des Parties à la Convention sur la diversité biologique a adopté le document dénommé « Les lignes directrices de Bonn $»^{24}$ par lesquelles elle a non seulement tenté d'harmoniser les législations nationales pour les pays pourvoyeurs de ressources, mais aussi a avancé des "mesures d'utilisateurs" afin de faire respecter les CLIP et le partage des avantages (Louafi : 165). Ainsi, ces négociations contribuent à renforcer les procédures et les «mesures d'utilisateurs » de brevets sur le vivant en aval de la " chaîne de valeur » plutôt que de focaliser les débats sur l'amont, dès lors que la détermination de la valeur des ressources naturelles, génétiques et des savoirs traditionnels associés reste très difficile.

Ces négociations, en aval, sur les " mesures d'utilisateurs ", relèvent alors d'un domaine où une nouvelle gouvernance s'impose au niveau international, obligeant à des mécanismes de transparence et de traçabilité des ressources biologiques (Louafi : 176) collectées dans les pays du Sud, qui n’ont pas d'équivalent jusque-là et qui laissent augurer que la lutte est encore longue. On peut

24. Lignes directrices de Bonn sur l'accès aux ressources génétiques et le partage juste et équitable des avantages résultant de leur utilisation. Sur les mesures d'utilisateurs, voir notamment le point II. C. 16.d, p. 7. PNUE-CDB. Disponible sur le site http://www. biodiv.org. 
cependant espérer que cette lutte aille vers une réduction des asymétries dans les rapports de force. Ainsi en est-il du " certificat de conformité » ou instrument de traçabilité qui oblige les détenteurs de brevets à indiquer l'origine des ressources prélevées, ce certificat constituant un outil qui « qui permettrait une meilleure vérification du rôle respectif des différents partenaires impliqués dans la chaîne de valeur » (idem).

Le Parc Amazonien de Guyane (PAG), à travers l'exigence dans sa charte des consentements libres, informés et préalables, montre qu'en amont aussi, l'accès aux ressources biologiques mobilise de manière plus systématique les acteurs qui se sentent concernés par la défense de leurs droits et menacés par les brevets. Les protocoles ADPIC, à condition qu'ils soient associés au respect des articles (8 et 15) de la CDB, doivent permettre aux peuples autochtones de Guyane, comme d'ailleurs, de renforcer leurs exigences, dès lors que ces mécanismes engagés et les dynamiques néolibérales de marchandisation du vivant restent une menace pour leur identité, leur culture, et leurs connaissances traditionnelles et, partant, pour la conservation de la biodiversité elle-même.

\section{Bibliographie}

AUBERTIN Catherine, « Transcription de la Convention sur la diversité biologique dans les législations nationales sur l'accès aux ressources génétiques (pays andins et Brésil). Tensions économiques et innovations sociales " (communication), Séminaire de l'IFB, Fréjus 7-9 septembre 2005, Fonds documentaire de l'IRD. Disponible sur http://horizon.documentation.ird.fr/exl-doc/ pleins_textes/divers09-09/010039776.pdf, consulté le 03/11/2014.

AUBERTIN Catherine \& Geoffroy FILOCHE, «Le Protocole de Nagoya sur l'utilisation des ressources génétiques: un jeu à somme nulle entre Nord et Sud? ", Mouvements, 17 mars 2011. Disponible sur http://www.mouvements. info/Le-Protocole-de-Nagoya-sur-l.htm, consulté le 22/09/2013.

AUBERTIN Catherine, PINTON Florence \& Valérie BOISVERT (sous la dir. de), Les marchés de la biodiversité, Paris, IRD Éditions, 2007, 269 p.

BERKES Fikret, "Environmental Philosophy of the Cree People of James Bay." Traditional Knowledge and Renewable Resource Management in Northern Regions. M. FREEMAN \& L. CARBYN (ed.) Edmonton: University of Alberta, Boreal Institute for Northern Studies, 1988. 7-21.

CHAPUIS Jean, La personne Wayana entre sang et ciel, tomes I \& II, thèse d'Anthropologie, Université d'Aix-Marseille, 1998, 1082 p.

CHAPUIS Jean \& Hervé RIVIĖRE, Wayana eitoponpë. (Une) histoire (orale) des Indiens Wayana, Matoury, Ibis rouge éditions, 2003, 1065 p. 
Congrès des élus de Guyane, Résolution du congrès pour l'accès aux ressources génétiques biologiques et aux savoirs associés et le partage des avantages, 21 juillet 2011.

DESCOLA Philippe, Par-delà nature et culture, Paris, Gallimard, 2006.

FILOCHE Geoffroy, «Les connaissances, innovations et pratiques traditionnelles en matière de biodiversité : un kaléidoscope juridique ", Droit et société $72: 2$ (2009) : 433-456.

FLEURENTIN Jacques, WENIGER B. \& G. BOURDY, Traditions thérapeutiques et médecines de demain. Les enjeux de l’ethnopharmacologie, Rennes, Éditions Ouest-France, 2011, $126 \mathrm{p}$.

FLEURY Marie, «Dénomination et représentation des végétaux en forêt tropicale : étude comparative chez les Amérindiens wayana et les Noirs marrons aluku de Guyane francaise ", Bahuchet, Serge, Bley, Daniel, Pagezy, Hélène \& Nicole Vernazza-Licht (sous la dir. de), L'homme et la forêt tropicale, Châteauneuf de Grasse, Édition de Bergier-SEH, 1999, 31-44.

" Du piment de l'agouti à la fiente d'aigle harpie : les animaux entre aliments, maux et remèdes chez les Wayana (Guyane française) », DOUNIAS, Edmond, MOTTE-FLORAC Elisabeth, MESNIL Marianne \& Margaret DUNHAM (sous la dir. de), Le symbolisme des animaux. L'animal clef-de-voûte dans la tradition orale et les interactions homme-nature/Animal symbolism. The "Keystone" Animal in Oral Tradition and Interactions between Humans and Nature, Paris, IRD, DVD, coll. « Colloques et Séminaires », 2007, 755-775.

FLEURY Marie, KARPE Philippe \& Alexis TIOUKA, « Valorisation des produits forestiers et construction d'un patrimoine guyanais : articulation entre le social, le biologique et le juridique ", MAM LAM FOUCK, Serge \& Isabelle HIDAIR (sous la dir. de), La question du patrimoine en Guyane, Matoury, Ibis rouge éditions, 2011, 191-215.

GREIBER Thomas, PEÑA MORENO Sonia, AHRÉN Mattias, NIETO CARRASCO Jimena, KAMAU Evanson C., CABRERA MEDAGLIA Jorge, \& Natasha ALI, An Explanatory Guide to the Nagoya Protocol on Access and BenefitSharing. Union Internationale pour la Conservation de la Nature : IUCN Library System, 2012. Disponible sur https://portals.iucn.org/library/node/10258, consulté le 03/11/2014.

GRENAND Pierre \& Françoise GRENAND, «Il ne faut pas trop en faire : Connaissance du vivant et gestion de l'environnement chez les Wayãpi (Amérindiens de Guyane) ». Cahier des Sciences Humaines 32.1 (1996) : 51-63.

GRAIN, Brevets, Pirates et Promesses perverties, Rapport, 2000. http:// www.grain.org/fr/article/entries/56-des-brevets-et-des-pirates. Consulté le 22/09/2013.

HOUDE Nicolas. "The Six Faces of Traditional Ecological Knowledge: Challenges and Opportunities for Canadian Co-Management Arrangements." 
Ecology and Society 12.2 (2007). Disponible sur http://www.ecologyandsociety. org/vol12/iss2/art34, consulté le 21/07/2014.

HUNKA Roger \& Joshua MCNEELY, Implementation of the Nagoya Protocol within Canada. Truro Heights: Maritime Aboriginal Peoples Council-Ikanawtiket Environmental Incorporated, 2012. Disponible sur http://www.ikanawtiket.ca/pdf/NagoyaProtocol-Eng.pdf, consulté le 03/11/2014.

JONAS Harry, BAVIKATTE Kabir \& Holly SHRUMM. "Community Protocols and Access and Benefit Sharing." Asian Biotechnology and Development Review 12.3 (2010): 49-76.

KARPE Philippe, Les collectivités autochtones, Paris, L’Harmattan, coll. « Logiques juridiques », 2008.

LOUAFI Selim, "Le certificat de conformité, un tournant majeur dans la gouvernance de l'accès et du partage des avantages ? ", NÈGRE Céline (sous la dir. de), La Convention Internationale sur la Biodiversité : enjeux de la mise en œuvre, Paris, La documentation française, 2010, 161-183.

MONOD Jacques, Le hasard et la nécessité. Essai sur la philosophie naturelle de la biologie moderne, Paris, Seuil, 1970.

MORETTI Christian \& Catherine AUBERTIN, «Stratégies des firmes pharmaceutiques : la bio-prospection en question ", AUBERTIN Catherine, PINTON Florence \& Valérie BOIVERT (sous la dir. de), Les marchés de la biodiversité, Paris, IRD éditions, 2007, 27-54.

MOTTE-FLORAC Elisabeth, "Quelques problèmes posés par l'ethnopharmacologie et la recherche pharmaceutique sur les substances naturelles ", Journal des anthropologues, 2002. Disponible sur http://jda.revues.org/2807 consulté le 22/09/2013, consulté le 22/09/2013.

Parc Amazonien de Guyane, "Projet de Charte », présenté au Conseil d'Administration le 30 août 2011, 2011, 159 p. + annexes.

PINTON Florence \& Pierre GRENAND, « Savoirs traditionnels, populations locales et ressources globalisées », AUBERTIN Catherine, PINTON Florence \& Valérie BOIVERT (sous la dir. de), Les marchés de la biodiversité, Paris, IRD éditions, 2007, 165-194.

Projet de Loi relatif à la biodiversité, $n^{\circ}$ 1847. République française. Ministère de l'écologie, du développement durable, et de l'énergie. Enregistré à la Présidence de l'Assemblée nationale le 26 mars 2014.

RATUVA Steven, "La marchandisation des savoirs culturels - La science occidentale au service des grandes entreprises et les savoirs autochtones du Pacifique ", Revue internationale des sciences sociales 1.195 (2010) : 179-190. Disponible sur http://www.cairn.info/revue-internationale-des-sciences-sociales2010-1-page-179.htm, consulté le 27/06/2014. 
THOMAS Frédéric, « Biodiversité, biotechnologies et savoirs traditionnels. Du patrimoine commun de l'humanité aux ABS (Access to Genetic Resources and Benefit-Sharing) », Revue Tiers-Monde 188 (octobre-décembre 2006) : 825-842.

WUNDER Sven, Payments for Environmental Services: Some Nuts and Bolts. Jakarta: Center for International Forestry Research, coll. "Occasional Paper $\mathrm{n}^{\circ} 42$ ", 2005, $24 \mathrm{p}$.

\begin{abstract}
Résumé : Les plantes médicinales sont perçues de manière très contrastée selon les différents protagonistes et usagers de la nature : pour les autochtones de Guyane elles font sens dans une approche spirituelle de la Nature, tandis que pour de nombreux autres acteurs, notamment dans le domaine pharmaceutique, elles sont perçues comme des usines à molécules bioactives, ou encore à travers leur potentiel économique. Après une description de ces différentes approches, et la manière dont elles se traduisent (ou pas) dans les textes élaborés pour protéger la biodiversité et les ressources génétiques (la CDB, le protocole de Nagoya, le dispositif d'Accès et de Partage des Avantages [APA]), leur mise en place en Guyane, le projet de loi sur la biodiversité, nous évoquerons le piège que représentent ces questions juridiques internationales pour les peuples autochtones mais aussi les dynamiques nouvelles qu'elles engendrent en termes de gouvernance pour l'ensemble des acteurs impliqués dans l'accès et le partage des avantages.
\end{abstract}

Mots-clés : Plantes médicinales, populations autochtones, savoirs traditionnels, Guyane française, ressources génétiques, projet de loi sur la biodiversité, Accès et Partage des Avantages (APA).

\begin{abstract}
Healing plants are perceived in very different ways according to various users of nature: for the Natives of French Guiana, they make sense in a spiritual approach of Nature, whereas for the other numerous actors, they are perceived as factories of bioactive molecules, or through their economic potential. After a description of these various approaches, and the way they are translated (or not) in texts developed to protect biological diversity and genetic resources (CDB, Nagoya protocol, protocols of access to the resources and sharing of the advantages) applied in Guiana's Region, French law's project on biodiversity, we discuss the trap posed by these legal questions for indigenous peoples, but also the new dynamics they generate in terms of governance for all stakeholders involved in Acces and Benefit Sharing (ABS).
\end{abstract}

Keywords: Healing plants, Indigenous people, traditional knowledge, French Guiana, genetic resources, French law on the biological diversity, Access and Benefit Sharing (ABS).

Laurence Boutinot est titulaire d'un doctorat en sociologie, Paris I Panthéon Sorbonne. Elle est depuis 1999 spécialiste en sciences sociales au service de l'environnement et de la société, au Cirad (Centre de coopération internationale en recherche agronomique pour le développement) à Montpellier, France. Elle a vécu pendant 15 ans en Afrique et a travaillé sur les questions scientifiques liées à la socio-anthropologie du changement social et du développement. Ses activités de recherche portent, entre autres, sur les questions d'approches participatives et de gouvernance locale, de décentralisation et de politiques publiques de l'environnement. Elle étudie ainsi les pratiques de gestion des ressources naturelles des populations autochtones et des communautés locales, les connaissances traditionnelles sur l'environnement, le genre et les pratiques culturelles dans les questions de développement.

Laurence Boutinot holds a Ph.D. in sociology, Paris I Pantheon Sorbonne. Since 1999, she has been a social scientist at the Environment and Society department, CIRAD in Montpellier, France. She lived for 15 years in Africa and worked on scientific issues related to Socio-anthropology of social change and development. Her research interests include issues of participatory approaches and local governance, decentralization and public policy for the environment. This implies studying management practices of natural resources of indigenous peoples and local communities, traditional knowledge 
about the environment, gender and cultural practices in development issues.

Marie Fleury, 53 ans, est ethnobotaniste et maître de conférence au Muséum National d'Histoire Naturelle depuis 1992. Diplômée en pharmacie, ethnologie et botanique tropicale, elle s'est spécialisée dans l'étude des relations entre l'homme et le milieu naturel en Guyane, en particulier sur le haut Maroni où elle poursuit ses recherches chez les Noirs marrons aluku et les Amérindiens Wayana depuis 1986. Auteure de plusieurs ouvrages et de nombreux articles, elle est impliquée localement dans la valorisation des savoir-faire traditionnels à travers la mise en place d'un circuit d'économie sociale et solidaire via une association loi 1901 (GADEPAM) regroupant plus de 300 artisans sur l'ensemble du territoire guyanais. Depuis 2007, elle dirige l'antenne du Muséum National d'Histoire Naturelle en Guyane.

Marie Fleury, age 53, is an ethnobotanist and lecturer at the National Natural History Museum since 1992. Awarded a diploma in pharmacy, ethnology and tropical botany, she specialized in the study of the relations between man and the natural environment in French Guiana, in particular on the upper Maroni river, where she pursues her research among aluku Maroons and Wayana Amerindians since 1986. Author of several works and articles, she is involved locally in the valuation of the traditional know-how through the implementation of a circuit of voluntary and united sector via a non-profit organization (GADEPAM) gathering more than 300 craftsmen on the Guyanese territory. Since 2007, she manages the antenna of the National Natural History Museum in French Guiana.

Philippe Karpe (philippe.karpe@cirad.fr) est chercheur en droit (Docteur-HDR) au CIRAD et un expert international sénior sur les droits des peuples autochtones. II mène actuellement une recherche avec Alexis Tiouka, expert juriste autochtone, sur le concept de citoyenneté autochtone en Guyane française.

Philippe Karpe (philippe.karpe@cirad.fr) is a Researcher in Law at the French Agricultural Research Centre for International Development (CIRAD) and a governmental expert on Indigenous peoples' law. He is currently studying the concept of Indigenous citizenship in the Guianese and French context. 